\title{
Coalesced and Embedded Nut Graphs in Singular Graphs
}

\author{
Irene Sciriha ${ }^{\dagger}$ \\ Department of Mathematics, Faculty of Science \\ University of Malta, Msida MSD 06, Malta
}

Received 1 August 2007, accepted 9 May 2008, published online 19 June 2008

\begin{abstract}
A nut graph has a non-invertible (singular) 0-1 adjacency matrix with non-zero entries in every kernel eigenvector. We investigate how the concept of nut graphs emerges as an underlying theme in the theory of singular graphs. It is known that minimal configurations (MCs) are necessarily found as subgraphs of singular graphs. We construct MCs having nut graphs as subgraphs. Nut graphs can be coalesced with singular graphs at particular vertices or grown into a family of core graphs of larger nullity by adding a vertex at a time. Moreover, we propose a local enlargement of nut fullerenes and tetravalent nut graphs.
\end{abstract}

Keywords: Adjacency matrix, nut graph, kernel eigenvector, singular graphs, core, periphery, nut fullerenes, line graphs of trees.

Math. Subj. Class.: 05C50, 05B20, 05C90, $92 E 10$

\section{Introduction}

A graph $G$ has vertex set $\mathcal{V}_{G}=\{1,2, \ldots, n\}$ and edge set $\mathcal{E}_{G}$ joining distinct vertices of $\mathcal{V}_{G}$. The complement $\bar{G}$ of $G$ has $\mathcal{V}_{\bar{G}}=\mathcal{V}_{G}$ and $\mathcal{E}_{\bar{G}}$ is the set of non-edges of $G$. The adjacency matrix $\mathbf{A}_{G}(=\mathbf{A}$ or $\mathbf{G})$, of a graph $G$, is the matrix $\left(a_{i j}\right)$, where $a_{i j}$ is one if $i j \in \mathcal{E}_{G}$ and zero otherwise. The graphs we consider are simple, i.e without loops or multiple edges; thus each entry on the main diagonal is zero. Since a graph is uniquely determined by its adjacency matrix, we use terminology for a graph and its adjacency matrix interchangeably. For different labellings of the vertices of a graph $G$, the corresponding adjacency matrices are similar. Thus the collection of eigenvalues $\lambda_{1}, \lambda_{2}, \ldots, \lambda_{n}$, (with repetitions), which is said to be the spectrum $S p(G)$ of $G$, is an invariant of $G[1,2]$.

\footnotetext{
${ }^{\dagger}$ Research supported by the AWRF, University of MALTA.

E-mail address: irene.sciriha-aquilina@um.edu.mt (Irene Sciriha)
} 
Singular graphs have the eigenvalue zero. In section 2, we survey properties of core graphs and nut graphs as particular singular graphs. Minimal configurations (MCs)and singular configurations (SCs) are subgraphs determined by the vectors in $\operatorname{ker}(\mathbf{A})$. A graph of nullity $\eta$ has $\eta$ independent MCs as subgraphs [17]. Catalogues of MCs of small order can be found in [11] and [13]. The simplest MCs are trees including paths on an odd number of vertices. A tree is a MC if and only if it is a subdivision of some tree [19]. In section 3, we survey the properties of MCs and proceed, in section 4, to explore possible ways of converting singular graphs to larger ones. If a graph $G$ has an eigenvalue $\lambda_{0}$ of multiplicity $k$, then $k$ vertices can be deleted to produce a star complement for $\lambda_{0}$, that is, a maximal induced subgraph of $G$ that does not have $\lambda_{0}$ as an eigenvalue. We show that a vertex deleted subgraph $Y-v$ of a nut graph $Y$ is a star complement for $\lambda_{0}=0$. Nut graphs can be coalesced with singular graphs at particular vertices to obtain larger nut graphs or other particular types of singular graphs. Moreover, if to nut graphs, vertices are added to increase the nullity at each stage, a family of core graphs of increasing nullity, with a nut graph as an induced subgraph, is produced. The last section presents a local enlargement for trivalent polyhedra, cylindrical constructions for nut fullerenes and an extension of four-valent polyhedra to produce larger core graphs.

\section{Nut Graphs}

A graph $G$ is singular if $\mathbf{A}$ is singular, that is if $\mathbf{A}$ is not injective. Hence, there exist linearly independent vectors $\mathbf{x}$, s.t. $\mathbf{A x}=\mathbf{0}$, so that zero is an eigenvalue of $\mathbf{A}$. The multiplicity $\eta$ of the eigenvalue zero is the dimension of the nullspace, $\operatorname{ker}(\mathbf{A})$ and is said to be the nullity $\eta(G)$ of $G$.

Definition 1. Let $G$ be a singular graph. A non-zero vector $\mathbf{x}$ satisfying $\mathbf{A x}=\mathbf{0}$ is said to be a kernel eigenvector of $G$.

There exist singular graphs, called core graphs, of nullity one or more, with a kernel eigenvector having no zero entries. We say that a subgraph, $F$, of a singular graph $G$, is a core of $G$, if there exists a kernel eigenvector x of $G$ such that $F$ is the subgraph induced by the vertices corresponding to the non-zero entries (or support) of $\mathbf{x}$. Hence a core $F$ of $G$ is a core graph, with a kernel eigenvector $\mathbf{x}_{F}$, the non-zero restriction of $\mathbf{x}$. The $|F|$ vertices of $F$ are determined by the $|F|$ labels corresponding to $\mathbf{x}_{F}$. We label a graph $G$, of nullity one, so that a kernel eigenvector of the form $\mathbf{x}=\left(\mathbf{x}_{\mathbf{F}}, \mathbf{0}\right)^{\mathbf{t}}$, generates the nullspace $\operatorname{ker}(\mathbf{G})$, where $\mathbf{X}_{\mathbf{F}} \in \mathbb{R}^{|F|}$ is the non-zero part of $\mathbf{x}$ with each of the $|F|$ entries being non-zero. For nullity greater than one, there are a number of cores, each corresponding to a non-trivial vector in $\operatorname{ker}(\mathbf{G})$. Note that each core of $G$ is a core graph in its own right.

The set of core vertices, $C V$, of $G$, consists of those vertices that lie in some core of $G$. A vertex, not lying in any core, is said to be a core-forbidden vertex. Thus a core graph has no core-forbidden vertices. Moreover, core graphs are distinguished from other singular graphs, since the deletion of any vertex reduces the nullity (Corollaries 13 and 16).

Definition 2. A nut graph is a singular graph such that no kernel eigenvector has zero entries.

Proposition 3. A nut graph is a core graph of nullity one.

Proof. By definition, a nut graph has at least one kernel eigenvector with no zero entries. Since linear combinations of linearly independent kernel eigenvectors can produce a kernel eigenvector with a zero entry and this cannot be the case for nut graphs, it follows that all 
kernel eigenvectors are linearly dependent. Hence, for a nut graph $Y$, the dimension of $\operatorname{ker} Y$ is one.

Theorem 4. [10] A nut graph

(i) is connected;

(ii) has noterminal vertex;

(iii) is not bipartite.

Remark 5. Nut graphs exist on seven or more vertices. The second and third graphs in Figure 1 are nut graphs. From Proposition 4(iii), it follows that all nut graphs have odd cycles. The girth of a graph is the size $i$ of the smallest odd cycle $C_{i}$, on $i$ vertices. It is given by the index $i$ of the first non-vanishing odd coefficient, $a_{i}$, in the characteristic polynomial $\sum_{i=0}^{n} a_{i} \lambda^{n-i}$ of the adjacency matrix of the graph [8]. There exist nut graphs with girth three. Nut fullerenes are three-regular and have girth five.

Proposition 6. [10] If a triangle $C_{3}=\left\{v_{1}, v_{2}, v_{3}\right\}$ is a subgraph of the nut graph $Y$ and the vertex $v_{1}$ is of degree two in $Y$, then $v_{2}$ and $v_{3}$ do not have the same neighbours. (Equivalently, $v_{2}$ and $v_{3}$ are not co-duplicate vertices.)

\section{Minimal Configurations}

In [14], we show that a singular graph of nullity $\eta$ has $\eta$ subgraphs called minimal configurations (MCs). In [17], a stronger result is obtained for singular configurations (SCs), namely that a singular graph of nullity $\eta$ has $\eta$ SCs as induced subgraphs. MCs and SCs are singular graphs of nullity one, determined by the non-zero part of their kernel eigenvector as follows $[11,17]$ :

Definition 7. Let $F$ be a core graph on at least two vertices, with nullity $s \geq 1$ and a kernel eigenvector $\mathbf{x}_{F}$ having no zero entries. If a graph $N$, of nullity one, having $\mathbf{x}_{F}$ as the nonzero part of a kernel eigenvector, is obtained, by adding $s-1$ independent vertices, whose neighbours are vertices of $F$, then $N$ is said to be a minimal configuration (MC).

The set of $s-1$ independent vertices added to $F$ to produce $N$ is said to be the periphery $\mathcal{P}(N)$ of $N$. Figure 1 shows three MCs with $s=2,1,1$ and $|\mathcal{P}(N)|=1,0,0$ respectively.

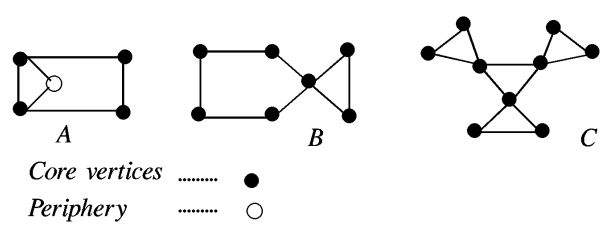

Figure 1: Three MCs

If the condition that the $(s-1)$ vertices added are independent is dropped, then the singular graph $S$ of nullity one obtained is said to be a SC. Various MCs may be constructed for the same $F$ and $\mathbf{x}_{F}$. The MCs, shown in Figure 2, have the same core $N_{5}=\overline{K_{5}}$ and $(1,1,-2,-1,2)$ or $(1,1,-1,-1,1)$ as the non-zero part of the kernel eigenvector.

We present properties of MCs. 


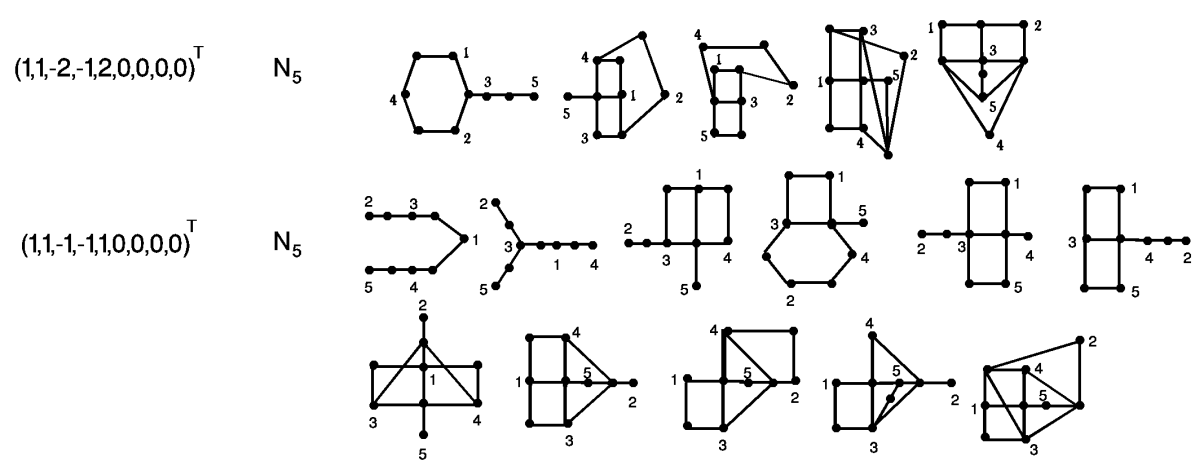

Figure 2: MCs with core $\overline{K_{5}}$ for two feasible 0 -eigenvectors

\section{Theorem 8. A MC is connected.}

Proof. Suppose, for contradiction that a MC $N$ is disconnected. Without loss of generality $N$ has a connected component $N_{1}$ having a non-zero kernel eigenvector $\mathbf{x}_{1}$. If the vertices of $N_{1}$ are labelled first, then $\mathbf{N}\left(\begin{array}{l}\mathbf{x}_{1} \\ \mathbf{x}_{2}\end{array}\right)=\left(\begin{array}{l}\mathbf{0} \\ \mathbf{0}\end{array}\right)$ and $\mathbf{N}_{1} \mathbf{x}_{1}=\mathbf{0}$. Since $\mathbf{x}_{1} \neq \mathbf{0}$, then $\mathbf{x}_{2}=\mathbf{0}$, otherwise $\left(\begin{array}{c}\mathbf{x}_{1} \\ \mathbf{0}\end{array}\right)$ and $\left(\begin{array}{c}\mathbf{0} \\ \mathbf{x}_{2}\end{array}\right)$ would be linearly independent kernel eigenvectors of $N$, contradicting that the nullity of $N$ is one. Thus $\mathbf{x}_{2}$ corresponds to vertices in the periphery and hence there are no edges among them. But then $N$ has isolated vertices which contribute to the nullity, so that $\eta(N)>1$, a contradiction. Thus $N=N_{1}$.

Theorem 9. [16] Let $N$ be a singular graph of order $n \geq 3$. The graph $N$ is a MC having a core $F$ with respect to the kernel eigenvector $\mathrm{x}$ and periphery $\mathcal{P}:=\mathcal{V}(N)-\mathcal{V}(F)$ if and only if the following conditions are all satisfied:
(i) $\eta(N)=1$,
(ii) $\mathcal{P}=\emptyset$ or $\mathcal{P}$ induces a graph consisting of isolated vertices,
(iii) $\eta(F)=|\mathcal{P}|+1$.

Proof. Condition (i) follows from the definition of MC.

If the nullity of $F$ is one, then $F=N$ and $\mathcal{P}=\emptyset$, so that both (ii) and (iii) are satisfied.

If the nullity $s$ of $F$ is more than one, then (ii) and (iii) follow from the construction of a $\mathrm{MC}$ in Definition 7.

Conversely, if the three conditions hold, then

a. $N$ has nullity one;

b. if $\mathcal{P} \neq \emptyset$, then the neighbours of the vertices in $\mathcal{P}$ are vertices of $F$;

c. by the Interlacing Theorem, condition (iii) requires that the nullity of the core $F$ decreases successively by one with each addition of the $(s-1)$ vertices in $\mathcal{P}$, in turn.

The above properties of MCs do not depend on the edges or non-edges among the vertices of the periphery, so that they hold also for SCs [17]. Note that a nut graph is a MC with $\mathcal{P}=\emptyset$. For $\eta(G)=1$, a singular graph $G$ has a unique core $F$ and a unique periphery. For $\eta(G)>1$, it is convenient to use a minimal basis $B_{\min }$ for the nullspace of $G$. The vectors 
$\mathbf{x}_{1}, \mathbf{x}_{2}, \ldots, \mathbf{x}_{\eta}$ in $B_{\min }$ are chosen so that the total number of non-zero entries in the vectors is a minimum. A somewhat surprising result is the following: if $\left|\mathbf{x}_{i}\right|$ denotes the number of non-zero entries in $\mathbf{x}_{i}$, then, every possible $B_{\min }$ has a unique sequence $\left|\mathbf{x}_{1}\right|,\left|\mathbf{x}_{2}\right|, \ldots,\left|\mathbf{x}_{\eta}\right|$ $[15,17]$.

\section{Extensions}

In [10], we explored the possibilities of growing nut graphs into larger nut graphs by adding vertices and modifying the kernel eigenvector. This prompts us to explore other ways of constructing singular graphs that have a nut graph embedded as an induced subgraph.

\subsection{Coalescence with Nut Graphs}

Definition 10. [9] If a vertex $v_{1}$ of a graph $G_{1}$ is identified with a vertex $v_{2}$ of a graph $G_{2}$, then the graph $G_{1} \cdot G_{2}$ obtained, of order $\left|G_{1}\right|+\left|G_{2}\right|-1$, is said to be the coalescence of $G_{1}$ and $G_{2}$.

We shall consider coalescence of singular graphs with nut graphs. The following theorem [9], which we shall have occasion to use, gives an expression for the characteristic polynomial $\phi(G, \lambda)$ of a graph $G=K . H$ in terms of characteristic polynomials of $K$ and $H$, and of their vertex-deleted subgraphs.

Theorem 11. The characteristic polynomial of the coalescence K.H of two rooted graphs (K,u), $(H, w)$ obtained by identifying the vertices $u$, and $w$ so that this vertex $v=u=w$ becomes a cut-vertex of $K . H$ is given by:

$$
\phi(K . H)=\phi(K) \phi(H-w)+\phi(K-u) \phi(H)-\lambda \phi(K-u) \phi(H-w) .
$$

Proposition 12. If $G$ is a graph of nullity $\eta$, there exists a subset $X$ of $\eta$ vertices of $\mathcal{V}(G)$, whose deletion yields a non-singular graph ${ }^{1}$.

Proof. Let $G$ be labelled so that the vertices in CV are labelled first. If $B=\left\{\mathbf{z}_{1}, \mathbf{z}_{2}, \ldots, \mathbf{z}_{\eta}\right\}$ is a basis for the nullspace of $\mathbf{A}(G)$, then the $\eta \times n$ matrix $\mathbf{M}$ whose rows are the $\eta$ vectors in $B$, has rank $\eta$. By row reduction, $\mathbf{M}$ can be reduced to Hermite Normal form $\mathbf{M}^{\prime}$, in which, to the (column) position of the first non-zero entry of each of the $\eta$ row vectors, there corresponds a zero entry in all the other rows. Since row reduction is equivalent to taking linear combinations of the kernel eigenvectors, the rows of $\mathbf{M}^{\prime}$ are also kernel eigenvectors of $G$. Deleting any vertex, $v$, affects just one of the row vectors so that the remaining $\eta-1$ rows of $\mathbf{M}^{\prime}$, restricted to $G-v$, are kernel eigenvectors of $G-v$. Moreover, there are no more kernel eigenvectors linearly independent of these $\eta-1$ row vectors as otherwise the nullity of $G$ would be more than $\eta$.

The following result follows from Proposition 12.

Corollary 13. If a core vertex $v$ is deleted from a graph $G$, of nullity $\eta$, then the nullity of $G-v$ is $\eta-1$.

The following result is also proved in [12], utilizing the adjugate of $\mathbf{A}$.

\footnotetext{
${ }^{1}$ For the eigenvalue zero, the graph $G-X$ is said to be a star complement of $G$ and the set $X$ a star set. See, for instance, [7].
} 
Corollary 14. If a core vertex $v$ is deleted from a singular graph $G$ of nullity one, then $G-v$ is non-singular.

Corollary 15. If any vertex $v$ is deleted from a nut graph $Y$, then $Y-v$ is non-singular.

Proof. This follows since $Y$ has nullity one and every vertex of $Y$ is a core vertex.

Corollary 16. If $\eta(G-v)<\eta(G)$ for all $v \in \mathcal{V}_{G}$, then $G$ is a core graph.

Proof. This follows since a basis for the nullspace of $\mathbf{A}(G)$ can be taken to be the row vectors of $\mathbf{M}^{\prime}$ as constructed in the proof of Proposition 12.

Proposition 17. A star set for the eigenvalue zero is contained in $C V$.

Proof. For the nullity to decrease on deleting a vertex $i$, there needs to be a kernel eigenvector in $M^{\prime}$ with its first non-zero entry at position $i$. These positions form a star set and are in CV by definition.

Proposition 18. If the graph $G^{\prime}$ is obtained by coalescing a nut graph $Y$ with a singular graph $G$, at a vertex $v_{F}$ of a core $F$ of $G$, then the nullity remains unchanged.

Proof. Let $\eta(G)=\eta$. Now by Corollaries 13 and 15, $\eta\left(G-v_{F}\right)=\eta-1$ and $\eta(Y-v)=0$. Thus $\eta\left(G^{\prime}\right) \leq \eta$ by interlacing.

From Equation (i) of Theorem 11, the characteristic polynomial $\phi\left(G^{\prime}\right)$ of the coalesence $G . Y$ is given by

$\phi\left(G^{\prime}\right)=\phi\left(G-v_{F}, \lambda\right) \phi(Y, \lambda)+\phi(G, \lambda) \phi\left(Y-v_{F}, \lambda\right)-\lambda \phi\left(G-v_{F}, \lambda\right) \phi\left(Y-v_{F}, \lambda\right)$. Each term on the right hand side of the equation is divisible by $\lambda^{\eta}$, so that $\eta\left(G^{\prime}\right) \geq \eta$. This completes the proof.

The construction of Proposition 18 may be done iteratively, running through all the core vertices, to produce successively larger graphs of the same nullity.

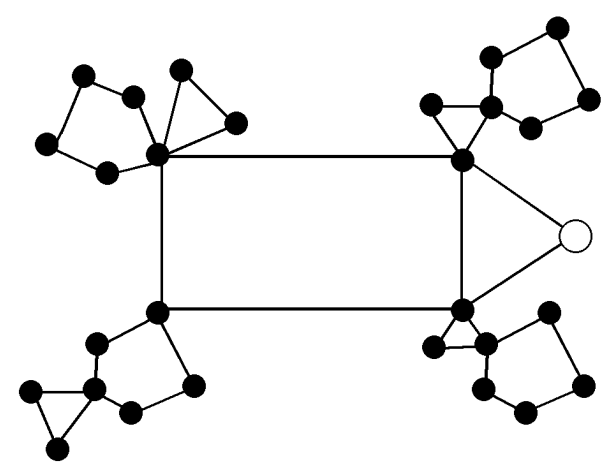

Figure 3: An enlarged MC

Figure 3 shows the graph $G^{\prime}$, an enlargement of the five vertex MC, $H$ (made up of $C_{4}$ and $C_{3}$ with a common edge). The smallest nut graph is the seven vertex, eight edge graph $Y$ of Figure 1, formed by coalescing $C_{5}$ with $C_{3}$. If $Y$ is coalesced, at each of the four core vertices of $H$, a graph $G^{\prime}$ is produced. By Proposition 18, $\eta(H)=\eta\left(G^{\prime}\right)$. 
Proposition 19. Let $N$ be a MC with core $F$. If nut graphs are coalesced with $F$ at distinct vertices of $F$, then a $M C$ is produced.

Proof. Let $G^{\prime}$ be constructed by coalescing nut graphs with $F$ at distinct vertices of $F$. At a vertex of $F$ where no nut graph is coalesced, we say that $K_{1}$ (the trivial nut graph) is coalesced with $N$. If $\mathbf{N}=\left(\begin{array}{cc}\mathbf{F} & \mathbf{B} \\ \mathbf{B}^{t} & \mathbf{0}\end{array}\right)$ represents the adjacency matrix of $N$, then, one way to produce the adjacency matrix of $G^{\prime}$, is to replace the diagonal entries of $\mathbf{F}$ in $\mathbf{N}$ by block matrices $\mathbf{Y}_{\mathbf{1}}, \mathbf{Y}_{\mathbf{2}}, \ldots, \mathbf{Y}_{|F|}$, where $Y_{i}$ is the nut graph (or $K_{1}$ ) coalesced at vertex $i$ for $1 \leq$ $i \leq|F|$, leave the principal submatrix for the $F$-vertices unchanged and insert zero matrices in the remaining blocks. Let $\mathbf{u}_{\mathbf{1}}, \mathbf{u}_{\mathbf{2}}, \ldots, \mathbf{u}_{|F|}$ be the eigenvectors of $Y_{1}, Y_{2}, \ldots, Y_{|F|}$, scaled so that the first entry of each $u_{i}$ is one. For $Y_{i}=K_{1}, \mathbf{Y}_{\mathbf{i}}=(0)$ and $\mathbf{u}_{i}=(1)$. If $\mathbf{N y}=\mathbf{0}$ and $\mathbf{y}=\left(y_{1}, y_{2}, \ldots, y_{|F|}, 0, \ldots, 0\right)^{t}$, then $\mathbf{G}^{\prime} \mathbf{z}=\mathbf{0}$, where

$\mathbf{z}=\left(y_{1} \mathbf{u}_{\mathbf{1}}{ }^{t}, y_{2} \mathbf{u}_{\mathbf{2}}{ }^{t}, \ldots, y_{|F|} \mathbf{u}_{|F|}^{t}, 0, \ldots, 0\right)^{t}$. Thus $G^{\prime}$ is singular with a core $F^{\prime}$, which is the coalescence of $F$ with $Y_{1}, Y_{2}, \ldots, Y_{|F|}$.

To show that $G$ is a MC, there remains to show that the three conditions of Theorem 9 are satisfied. Indeed, by Proposition 18, iterative coalescence at core vertices of $N$ preserves the nullity at the value one, so that condition (i) is satisfied. The periphery $\mathcal{P}^{\prime}=\mathcal{V}\left(G^{\prime}\right) \backslash \mathcal{V}\left(F^{\prime}\right)$ is the same independent set as $\mathcal{P} F=\mathcal{V}(G) \backslash \mathcal{V}(F)$, satisfying condition (ii). Also, by Proposition 18, $\eta\left(F^{\prime}\right)=\eta(F)$. Thus, deleting all the vertices of the periphery in turn from $G^{\prime}$ has the same effect on the nullity of $G^{\prime}$ as deleting the corresponding vertices of $\mathcal{P}$ in $G$, so that the nullity increases at each stage, as required for condition (iii). Hence $G^{\prime}$ is also a MC.

Remark 20. Since the construction in Proposition 19 does not affect the vertices in the periphery, the following result is immediate.

Corollary 21. Let $Y_{1}$ and $Y_{2}$ be nut graphs. The coalescence $Y_{1} . Y_{2}$ is a nut graph.

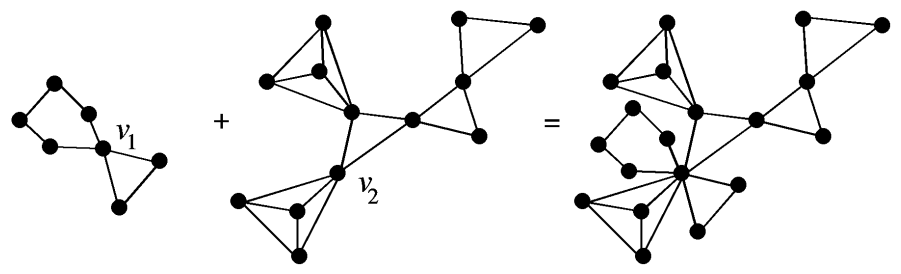

Figure 4: The coalescence of two nut graphs.

In Figure 4, the nut graph on 7 vertices is coalesced with the nut graph on 15 vertices, at arbitrary vertices $v_{1}$ and $v_{2}$, to produce a larger nut graph.

\subsection{Singular Graphs with a Nut Graph Embedding}

Duplicate vertices are independent vertices having the same neighbours. They correspond to identical rows (and columns) in A. A set of $\ell$ duplicate vertices contributes $\ell-1$ to the nullity of a graph. A kernel eigenvector corresponding to a core graph consisting of a pair of duplicate vertices has two non-zero entries only. A vertex can always be added to form a duplicate pair with any vertex of a graph, thus increasing the nullity indefinitely. Following 
Lepović, we call a graph canonical, if it has no duplicate vertices [5]. The kernel eigenvectors of a singular canonical graph have three or more non-zero entries.

The Reconstruction Theorem [6,7] applied to the eigenvalue zero states that a finite number, $k$, of vertices are added to a zero-star complement to produce a canonical supergraph of maximal nullity $k$. By Corollary 15, a vertex-deleted subgraph of a nut graph is a zero-star complement.

Proposition 22. If a singular graph $G$ of nullity $\eta$ is obtained by adding $\eta-1$ vertices to a nut graph $Y$, then $G$ is a core graph.

Proof. Let $B_{1}$ be the basis containing only the nullspace generator of $Y$. Assume that $j-$ 1 vertices have been added to $Y$ to produce $G_{j}$ with nullity $j, j \geq 1$ and the basis $B_{j}$, containing $j$ vectors, generates the nullspace of $G_{j}$. If a vertex $v$ can be added to increase the nullity by one, a kernel eigenvector $\mathbf{x}_{\mathbf{j}+\mathbf{1}}$, linearly independent of the ones in $B_{j}$, is included to produce $B_{j+1}$. We can proceed inductively to produce a larger singular graph $G_{\eta}$ with a basis $B_{\eta}$, by adding to $B_{1}$, vectors having a non-zero entry in positions $|Y|+j-1$ corresponding to the additional vertices in turn, for $0 \leq j \leq \eta-1$. Note that an entry of $\mathbf{x}_{\mathbf{j}+\mathbf{1}}$ in the position corresponding to $v$ is necessarily non-zero. Thus the graph $G_{\eta}$ is a core graph.
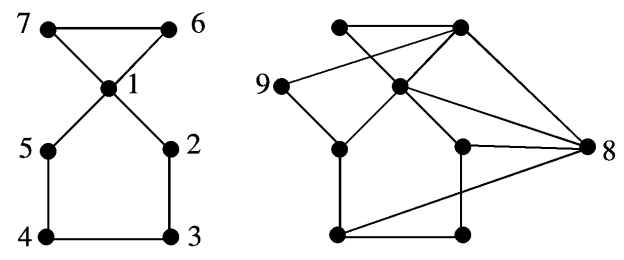

Basis for the nullspace

$(-1,-1,1,1,-1,1,1,0,0)$

$(-1,0,1,0,-1,1,0,0,1)$

$(-1,-1,0,1,-1,1,0,1,0)$

Figure 5: A core graph of nullity three with a nut graph embedded.

In Figure 5, to the nut graph on 7 vertices, two vertices can be added to produce a singular canonical graph of nullity three. The two graphs produced in the process are core graphs.

\section{Nut Fullerenes}

We conclude with a reference to the significance of nut graphs in chemistry, presenting ways of transforming nut regular polyhedra to larger core graphs. The Húckel Molecular Orbital (HMO) model describes the distribution of the $\pi$-electrons in unsaturated organic molecules and is an approximation to the solution of the Schrődinger Equation [4]. The molecular graph $G$ of a Carbon (C) structure has the $\mathrm{C}$-atoms as vertices and the $\mathrm{C}-\mathrm{C} \sigma$ bonds as edges. The energy levels $\lambda_{i}$ of the $\pi$-electrons, in the C-structure, are given by $\mathbf{A} \mathbf{x}_{i}=\lambda_{i} \mathbf{x}_{i}$, where $\mathbf{x}_{i}$ describes the molecular orbital for the energy $\lambda_{i}$ and $\mathbf{A}$ is the adjacency matrix of $G$. For singular molecular graphs, $\mathbf{A x}=\mathbf{0}, \mathbf{A}$ has zero eigenvalues, and the $\mathrm{C}$-structure has nonbonding molecular orbitals (NBMOs) described by $\mathbf{x}(\neq \mathbf{0})[4]$.

If $G$ is a nut graph, then the NBMO x of the $\pi$-electron occupying this orbital has nonzero entries. In chemistry, this is significant. In the radical molecule that would have the NBO as partially occupied HOMO, all centres carry non-zero spin density. The charge, spin and bond-order densities of a $\pi$-electron, occupying the NBMO, are distributed over 
the whole structure and not on some substructure. Thus all C-centres are likely to be involved in reactions. $[18,20,21]$.

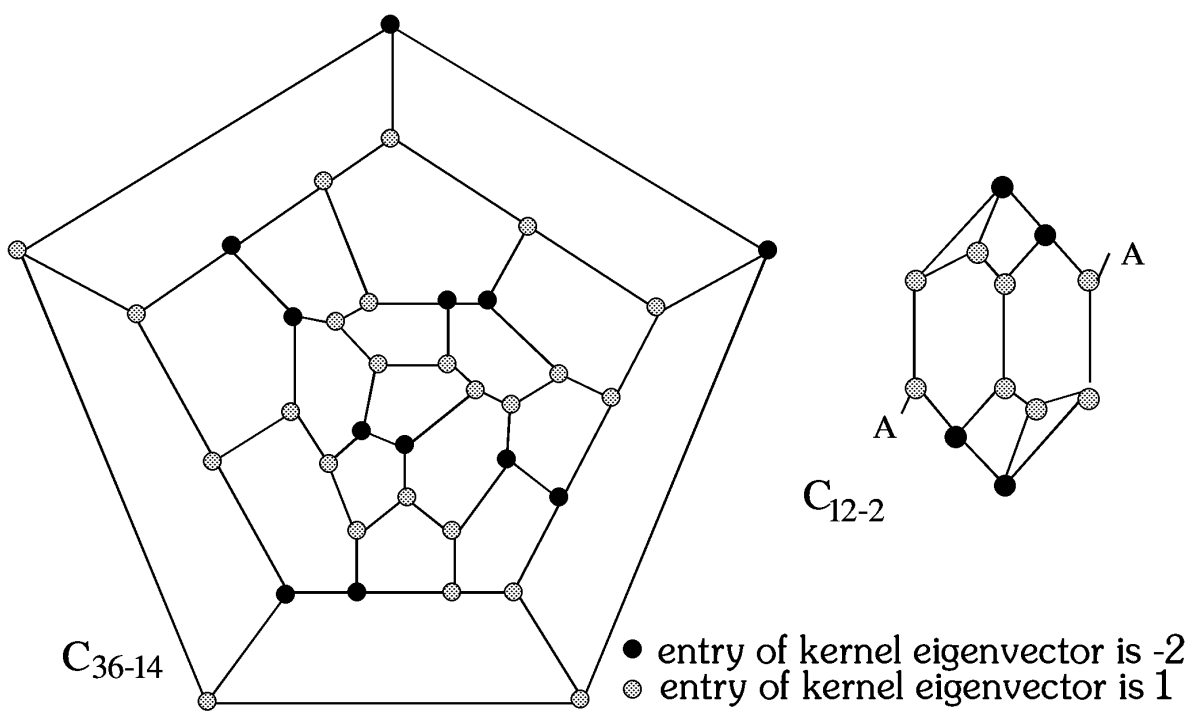

Figure 6: $\mathrm{C}_{36}: 14$ and $\mathrm{C}_{12}: 2$

There are 10, 190, 782 fullerene isomers on up to 120 vertices and only 41 are nut graphs. In all hypothetical nut fullerenes found so far, occupation of the NBMO requires that the fullerene carries at least five negative charges. All physically realizable fullerenes, known to date, have isolated pentagons and none of these is a nut graph.
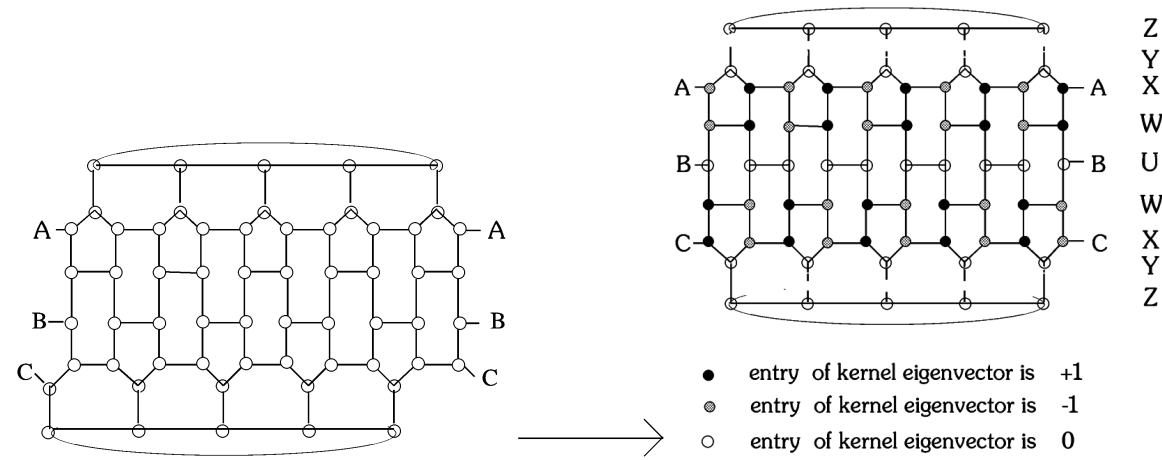

- entry of kernel eigenvector is +1

- entry of kernel eigenvector is -1

- entry of kernel eigenvector is 0

Figure 7: $\mathrm{C}_{60}$ and $\mathrm{C}_{70}$

The smallest nut fullerene is $C_{36}: 14$ [3] and the smallest nut trivalent polyhedron is $C_{12}: 2$, both shown in Figure 6 . Symmetry studies of polyhedra yield powerful mathematical results that can be immediately translated into chemical properties $[18,20]$. For highly 
symmetric vertex transitive or one-orbit graphs, the eigenvector entries for a simple eigenvalue, have the same non-zero absolute value. This implies equidistributivity of charge from the single NBMO of a molecule. However $\mathbf{A x}=\mathbf{0}$, with each entry of $\mathbf{x}$ being \pm 1 , demands an even number of bonds for each $\mathrm{C}$-centre which is not the case in tri-valent polyhedra. Thus vertex transitive nut fullerenes are ruled out. Several trivalent $(+1,-1,0)$ molecules with a NBMO are possible. One is $C_{70}$ which can be seen as the Buckminster fullerene $C_{60}$ with an additional ten $\mathrm{C}$-atoms, contributing an equatorial ring of five extra hexagons, as shown in Figure 7. Such a singular trivalent structure cannot be a MC, since every C-centre (even if it corresponds to a zero entry of the kernel eigenvector) has an adjacent atom with no charge from the NBMO. This forces two peripheral vertices to be adjacent, a configuration which is not allowed in MCs.

The two nut trivalent polyhedra $C_{36}: 14$ and $C_{12}: 2$ mentioned above have a $(-2,+1$, $+1)$ NBMO, that is a kernel eigenvector with $-2,+1$ as its only entries. Such graphs are said to be uniform nut graphs. Considering orbits, since every C-centre is adjacent to three atoms corresponding to entries $-2,+1$ and +1 , one can deduce that the number of $\mathrm{C}$ atoms in uniform-nut fullerenes is a multiple of six [18, 20, 21].

Inspection of the pictures and spiral codes of the early nut fullerenes suggests some recurring patterns. We now investigate ways in which nut chemical graphs can be expanded, while the original part of the nullspace generator remains in the new nullspace.

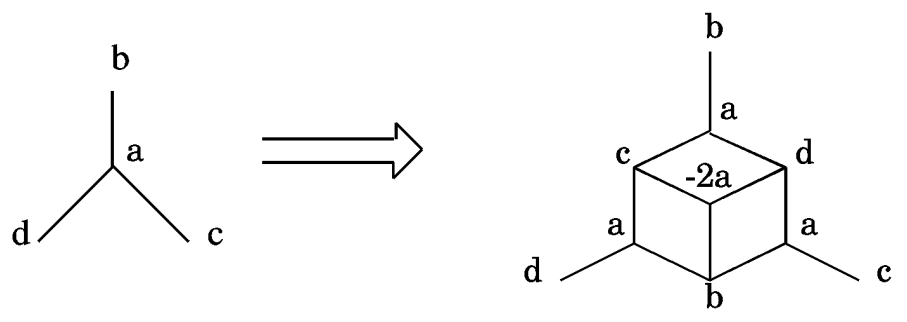

Figure 8: Local enlargement in regular chemical graphs

To preserve the regularity of a trivalent graph, coalescence cannot be employed. Instead an expansion of cubic graphs that preserves the null eigenvector can be considered. The following construction was suggested by P.W. Fowler. In a kernel eigenvector, the central vertex carries entry $a$ and by applying the zero-sum rule (or eigenvector equation for the zero eigenvalue) the entries on its neighbours total $b+c+d=0$. Expansion of the central vertex to a half-cube, as shown in Figure 8, can be achieved without disruption of the null eigenvector, if new eigenvector entries are assigned as shown. Clearly, if the parent graph is a uniform nut graph and $a=+1$, the derived vector is uniform, but if $\left.a=(-2)^{r-1}, r \geq 3\right\}$, then the entries of the derived vector are in $\left\{(-2)^{r-1}, r \geq 1\right\}$.

As the vertex count increases, cylindrical nut fullerenes occur, and can be grouped into families according to their six-pentagon caps. In [21], it is shown that there exists a family of tubular fullerenes, each with a cap of six pentagons and two hexagons, circumscribed by successive rings of six hexagons and ending with the same cap, which have a kernel eigenvector with entries in $\{1,-2\}$. The pattern of coefficients is compatible with insertion of any number of extra layers of six hexagons in the starting $\mathrm{C}_{48}$ structure, so that we have an infi- 


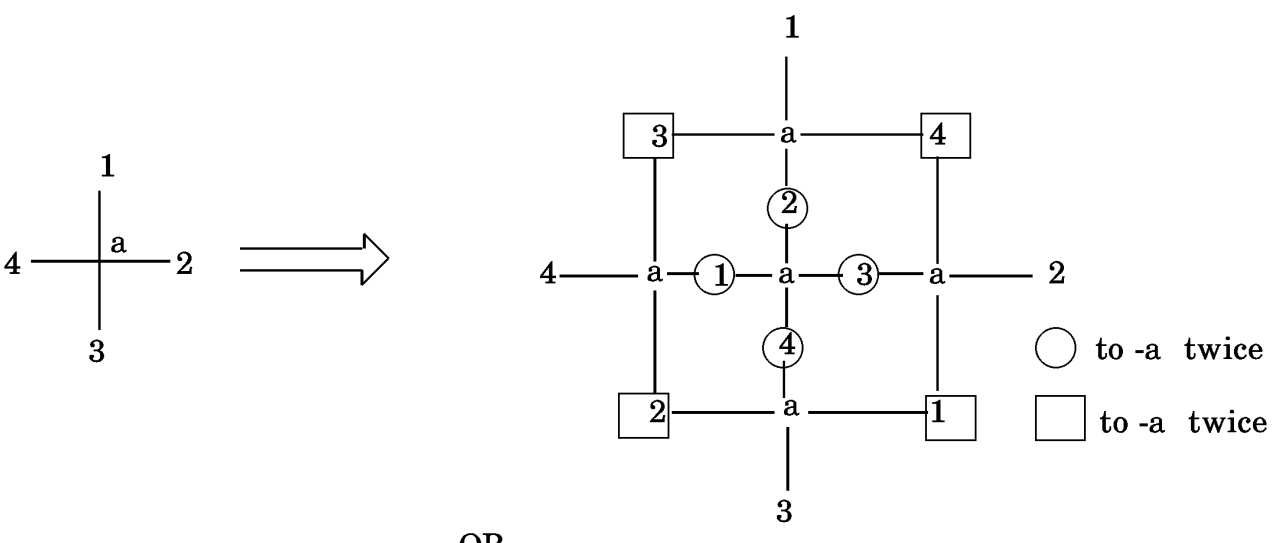

OR

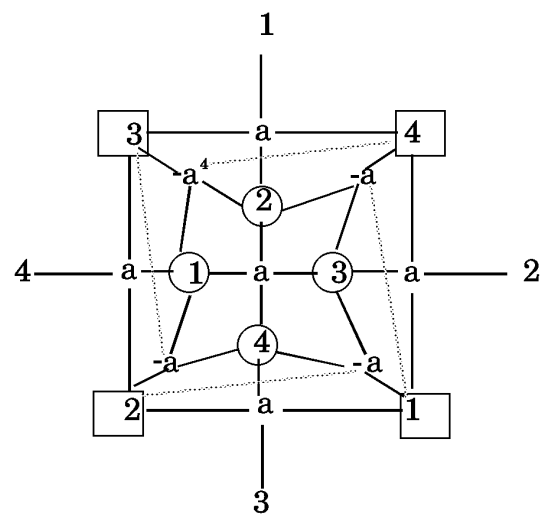

Figure 9: Local enlargement in trivalent chemical graph.

nite series of singular fullerenes, with predictable spiral codes. All members of the series are cores. Although we have no formal proof that the multiplicity of the zero eigenvector remains at the value one after each stage of the expansion, the null eigenvector in each member of the series is found to remain unique at least for all $n \leq 1000$, and it appears plausible that we have an infinite family of not only core fullerenes but also uniform-nut fullerenes.

We propose two similar expansions of tetravalent molecules where, in a kernel eigenvector, the central vertex carries entry $a$ and the zero-sum rule necessitates that the addition of the four neighbouring entries be zero. The bonds (edges) for the added atoms (vertices) are shown in Figure 9. Note that in the first construction, two additional vertices, each carrying charge $-a$, one above and one below the plane of the diagram, are required to produce a four-valent molecule which is a core graph. In the second construction all the edges are shown.

\section{References}

[1] D. Cvetković and I. Gutman, Algebraic Multiplicity of Zero in the Spectrum of Bipartite Graphs, Mat. Vesnik (Beograd) 9 (1972), 141-150. 
[2] D. M. Cvetković, P. Rowlinson and S. Simić Eigenspaces of Graphs, Encyclopedia of Mathematics and its Applications 66, Cambridge University Press, Cambridge, 1997.

[3] P. W. Fowler, P. W. Manolopoulos, An Atlas of Fullerenes, Clarendon Press, 1995.

[4] E. Hückel, Zur modernen Theorie ungesättigter und aromatischer Verbindungen, Z. Elektrochem. 61 (1957), 866-890.

[5] M. Lepović, On Canonical Graphs and Eigenvectors of Graphs, Utilitas Mathematica 54 (1998), 257-272.

[6] P. Rowlinson, Star Sets and Star Complements in Finite Graphs - A Spectral Construction Technique, Publications de L'Istitut Mathématique, Nouvelle Série 76 (90) (2004), 25-30.

[7] P. Rowlinson, Star Complements in Finite Graphs - A Survey, Rendiconti del Seminario Matematico di Messina Ser II Supplimento al n. 8 (2002), 145-162.

[8] H. Sachs, Besiehungen Zwischen den in Einem Graphen Enthaltenen Kriesen und Seinem Charakteristischen Polynom, Publ. Maths. Debrecen 11 (1964), 261-274.

[9] A. J. Schwenk, Computing the Characteristic Polynomial of a Graph, in: R. A. Bari and F. Harary, eds., Graphs and Combinatorics 406, Springer-Verlag, 1975, 153-172.

[10] I. Sciriha and I. Gutman, Nut Graphs - Maximally Extending Cores, Utilitas Mathematica 54 (1998), 257-272.

[11] I. Sciriha, On the Construction of Graphs of Nullity One, Discrete Math. 181 (1998), 193-211.

[12] I. Sciriha, On the Coefficient of $\lambda$ in the Characteristic Polynomial of Singular Graphs, Utilitas Mathematica 52 (1997), 97-111.

[13] I. Sciriha, On the Rank of Graphs, in eds. Y. Alavi et al., The Eighth Quadrennial International Conference on Graph Theory, Combinatorics, Algorithms and Applications, Springer-Verlag, 1998, 769-778.

[14] I. Sciriha, On some Aspects of Graph Spectra, Ph.D. Thesis, University of Reading, UK, British Library, November 1998.

[15] I. Sciriha, S. Fiorini and J. Lauri, A Minimal Basis for a Vector Space, Graph Theory Notes of New York XXXI 1996, 21-24.

[16] I. Sciriha and I. Gutman, Minimal Configurations and Interlacing, Graph Theory Notes of New York XLIX (2005), 38-40.

[17] I. Sciriha, A Characterization of Singular Graphs, Electronic Journal of Algebra 16 (2007) 451462.

[18] I. Sciriha and P. W. Fowler, A Spectral View of Fullerenes, Mathematica Balkanica 18, Fasc. 1-2, (2004), 183-192.

[19] I. Sciriha and I. Gutman, Minimal Configurations Trees, Linear and Multilinear Algebra, 54-2 (2006), 141-145.

[20] I. Sciriha and P. W. Fowler, Nuts, Cores and Symmetry in Fullerenes, Discrete Math. 308 (2008), 267-276.

[21] I. Sciriha and P. W. Fowler, Non-bonding Orbitals in Fullerenes: Nuts and Cores in Singular Polyhedral Graphs, Journal of Chemical Information and Modeling, J. Chem. Inf. Model 47 (2007), 1763-1775. 